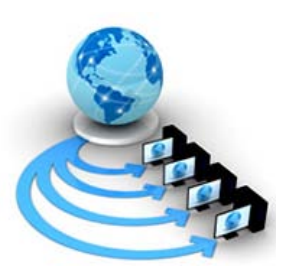

Volume 8, No. 8, September-October 2017

International Journal of Advanced Research in Computer Science

RESEARCH PAPER

Available Online at www.ijarcs.info

\title{
EXPLOITING NOISE AND TEXTURAL FEATURES FOR PASSIVE IMAGE FORENSICS
}

\author{
Surbhi Gupta \\ Research Scholar \\ K. Gujral Punjab Technical University \\ Kapurthala, India,
}

\author{
Neeraj Mohan \\ Assistant Professor \\ K. Gujral Punjab Technical University, \\ Kapurthala, India,
}

\author{
Parvinder Singh Sandhu \\ Professor, CSE, \\ India
}

\begin{abstract}
Image forensics is an emerging research domain which deals with detecting image forgeries. It authenticates images which are suspected of being manipulated. The authenticity of an image has become challenging these days as a lot of image manipulation tools are available. This research paper aims to introduce an integrated approach to detect image tampering. The proposed approach exploits noise and texture features to classify authentic and tampered images using support vector machine. Twenty Four statistical features are extracted and are used for classification. Proposed method has been evaluated and compared with existing methods. Experimental results demonstrate that proposed method gives good accuracy with low dimensionality of features.
\end{abstract}

Keywords: Image forensics is an emerging research domain which deals with detecting image forgeries

\section{INTRODUCTION}

Images play an important role in present digital era. Much information is communicated using images. Wide usage of images has led to their intended manipulation. Manipulation or tampering is usually done to change the content of the image. Such tampering in image is known as image forgery (Rocha, 2011). This research paper aims to introduce a combined approach to detect image tampering. The proposed approach exploits noise and texture features to classify authentic and tampered images using support vector machine. Tampered images are usually generated by using two or more images. This is known as image splicing (Birajdar, 2013). The techniques used for identifying forged images are known as image forensics. The forensic techniques utilize the image information to decide whether the questioned image is authentic or forged. Some of these techniques try to fix the source of the image, other try to compare it with some referenced images while other extract features to check inconsistencies present in image itself. This paper aims to present a passive classifier based on noise discrepancies and texture information present in the forged image.

Various image forensic techniques are worked upon by researchers. Xin (2002) proposed a noise based blind passive image quality assessment model to transform the heuristics into noise structures. Avcibas et al. (2004) proposed image forensic tool to expose compression and steganography using statistical analysis of image quality measures. Further Popescu and Farid (2004) suggested the noise variance of overlapping blocks from the image is used to estimate manipulation. Bayram et al. (2006) introduced Binary Similarity Measures (BSM) based on correlation and texture characteristics between the bit planes. Gou et al. (2009) in his paper proposed statistical noise feature extraction using de-noising, wavelets and likelihood of neighbors. Zhang et al. (2008) proposed splice detection based on measuring statistical differences using multi-size block discrete cosine transform and image quality measures. Mahdian and Saic (2009) performed block wise wavelet analysis followed by tiling sub band HH1and noise variance estimation for detecting manipulations. Wang et al. (2009) compared features from chroma and RGB channels for edge sensitivity and sharpness of inserted objects. Battisti et al. (2012) used blocking, Activity and Zero crossing characteristics from YCbCr channels for image highlighting tampered area in manipulated images. Later, Ke et al. (2014) proposed variance based noise estimation in forged images using PCA from HSV channels. Recently, Liu and Pun (2015) in used Sobel operator to detect sharp edged areas of foreign objects to localize the manipulated regions in the image. Many classifiers based on Natural Model (Shi, 2007), Markov features (He, 2012), Gabor filters (Muhammad, 2014), Multi Scale Weber Local Descriptors (Hussain, 2015) and Textural features (Shen, 2016) have been utilized for image forensics with good accuracy but high dimensionality.

\section{METHODOLOGY USED}

The forged images may be produced by copy move, splicing or various other post processing operations. Proposed method is based on statistical estimation of image noise introduced as a result of these operations. It is based on extracting image features from various color channels. Every color model highlights different set of features of an image. Depending on various color models we can extract much useful information which can be utilized to categorize authentic and tampered images. The used feature set is extracted using statistical noise and texture features from 
RGB and YCbCr planes of the image. A total of 24 features are extracted.

\section{NOISE BASED STATISTICAL FEATURES EXTRACTION}

Manipulations in images usually introduce some noise in the image. If this noise is detected and studied one could get clues for tampering. Noise based features could play a significant role in differentiating direct camera images from their tampered version. In this paper we are using statistical noise features to differentiate tampered images from original ones. De-noising algorithm to extract the image features has been employed. Various noise filters are capable of capturing different aspects of the image. Basically all of them are used for blurring and remove details and noise from images. Averaging, Median, Gaussian and Wiener filter of size 3X3 are considered. Averaging filter filters the image by taking averages of intensity values of the neighbor pixels as

$$
G(x, y)=\frac{1}{m n} \cdot \sum_{\mathrm{i}=1}^{\mathrm{m}} \sum_{\mathrm{j}=1}^{n} \mathrm{~F}(\mathrm{x}, \mathrm{y})
$$

A Median Filter filters the image by taking the median of intensity values of the neighbor pixels.

$$
G(x, y)=\operatorname{median}\{F(m, n)\}
$$

Where, $(m, n) \in w$, and $\quad w r e p r e s e n t s$ a neighborhood defined by the user, centered around location $x, y$ in the image. Gaussian filtering is used to blur images and remove noise and detail. Gaussian low pass filter with standard deviation 0.5 is taken in this work.

$$
G(x, y)=\frac{1}{2 \pi \sigma^{2}} \cdot e^{-\left(x^{2}+y^{2}\right) / 2 \sigma^{2}}
$$

Where, $\mathrm{x}$ is the distance from the origin in the horizontal axis, $y$ is the distance from the origin in the vertical axis, and $\sigma$ is the standard deviation of the Gaussian distribution. Weiner Filter filters the image to estimate the local image mean and standard deviation.

$$
G(x, y)=\mu+\frac{\sigma^{2}-v^{2}}{\sigma^{2}} \cdot F(x, y)-\mu
$$

Where, $\mu$ and $\sigma^{2}$ estimates the local mean and variance around each pixel and $v^{2}$ denoted noise variance. Mean and standard Deviation as in Eq. (1)-(2) are used as features for classification.

$1^{\text {st }}$ feature: $\mu=\frac{1}{M N} \sum_{i=1}^{M} \sum_{j=1}^{N} D_{R}(x, y)$

$2^{\text {nd }}$ feature: $\sigma=\frac{1}{M N} \sum_{\mathrm{i}=1}^{\mathrm{m}} \sum_{\mathrm{j}=1}^{\mathrm{n}}\left(\mathrm{D}_{\mathrm{R}}(x, y)-\mu\right)^{2}$

Four different types of filters for ' $\mathrm{R}$ ' channel are used. Thus, a total of $4 \times 2=8$ features are used.

\section{TEXTURE BASED STATISTICAL FEATURES EXTRACTION}

Apart from noise discrepancies the change texture of an image can also contribute in capturing the image details. Whenever as image is manipulated, the texture of manipulated areas differ from original ones. Especially the edges of introduced regions will have texture difference. Edges of objects present in image can reveal their nature.
The idea is to study edge texture features and then utilize them to identify tampering. In this paper, we utilized chroma ' $\mathrm{Cb}$ ' channel for extracting edge texture features from images. $\mathrm{Y}, \mathrm{Cb}$ and $\mathrm{Cr}$ channels are able to identify the sharpness of edged created due to copy move and splicing. First edge images are extracted using Sobel, Canny, Prewitt and LoG detectors.

After applying edge detector, gray-level co-occurrence matrix (GLCM) is created for each edge image to specify the pixel pair occurrences. GLCM can be calculated for different directions and offset. Only the right neighbor of the pixel is used in present work. Further contrast, energy, correlation and homogeneity as in Eq. (3)-(6) are considered for each edge operator. Contrast will measure the confined variations in the gray-level co-occurrence matrix. Correlation gives the joint probability occurrence of specific pixel pairs. Energy gives the sum of squared elements in the GLCM. Homogeneity denotes that how closely the elements in the GLCM are distributed to the GLCM diagonal.

$1^{\text {st }}$ feature:Energy $=\sum_{\mathrm{i}=0}^{\mathrm{G}-1} \sum_{\mathrm{j}=0}^{\mathrm{G}-1}\left(\mathrm{P}_{\mathrm{Y}}(i, j)^{2}\right.$

$2^{\text {nd }}$ feature: Correlation $=\sum_{\mathrm{i}=1}^{\mathrm{G}-1} \sum_{\mathrm{j}=0}^{\mathrm{G}-1} \frac{\{\mathrm{i}-\mathrm{j}\} \cdot \mathrm{P}_{\mathrm{Y}}\left((\mathrm{i}-\mathrm{j})-\left\{\mu_{\mathrm{x}}-\mu_{\mathrm{y}}\right\}\right.}{\sigma_{\mathrm{X}} \mathrm{X} \sigma_{\mathrm{y}}}(4)$

$3^{\text {rd }}$ feature: Contrast $=\sum_{\mathrm{n}=0}^{\mathrm{G}-1} \mathrm{n}^{2}\left\{\sum_{\mathrm{i}=1}^{\mathrm{G}} \sum_{\mathrm{j}=1}^{\mathrm{G}} \mathrm{P}_{Y}(\mathrm{i}, \mathrm{j})\right\}$

$4^{\text {th }}$ feature:Homogeneity $=\sum_{\mathrm{i}=0}^{\mathrm{G}-1} \sum_{\mathrm{j}=0}^{\mathrm{G}-1} \frac{1}{1+(\mathrm{i}-\mathrm{j})} \cdot \mathrm{P}_{\mathrm{Y}}(\mathrm{i}, \mathrm{j})$

A total of $4 \mathrm{X} 4=16$ features were used for classification.

\section{EXPERIMENTAL RESULTS}

The experimental results for the proposed method have been obtained using a popular image dataset CASIA V2.0 (Dong, 2011) .This dataset consists of 7,491 authentic and 5123 tampered images. Tampered images are produced using crop-paste and spliced image region(s). These images are pre- processed with resizing, rotation or other distortions. Images are post-processed with operations such as blurring to finish crop-and-paste operations. Different size (small, medium and large) of spliced regions has been considered. A $n$ fold cross validation approach is used to train and test the support vector machine for the proposed method. This approach divides the dataset in $n$ subsets. Then, $(n-1) / n$ proportion of the set is taken as training and $1 / n$ proportion is taken as testing set. Most of the state of art methods use 10 folds cross validation. So, we experimented for 10 fold cross validation to evaluate the performance of proposed method. LIBSVM (Chang, 2011) classifier with Radial Basis Function kernel is used, as it is well known for its performance for binary classification. The penalty parameter C is chosen by Grid Search method.

Accuracy of a classifier determines the correct classification of images by the classifier. It is observed that classification accuracy is better when authentic and forged images are at different quality factors. Proposed classifier is further compared with existing State of art techniques. Accuracy, dimensionality and need for feature selection are compared and tabulated in Table 1. Dimensionality means the number of features used for classification. Lesser dimensionality means less features and less evaluation time. Feature selection may be further used in some methods and it leads to increased evaluation time. 
Table 1 Performance comparison with existing methods

\begin{tabular}{|l|c|c|c|c|}
\hline Parameters & Proposed method & $\begin{array}{c}\text { Natural image } \\
\text { model }\end{array}$ & Markov features & Textural feature \\
\hline Accuracy & 94.56 & 84.86 & 89.76 & 97.73 \\
\hline Dimensionality & 24 & 266 & 100 & 96 \\
\hline Need for feature selection & Not Required & Not Required & Required & Not Required \\
\hline
\end{tabular}

It is evident that accuracy of proposed method is higher than natural model (Shi, 2007) and Markov features (He, 2012) with less dimensionality. Moreover, feature selection is not required for proposed method. Textural feature based method (Shen, 2016) has higher accuracy but has higher dimensionality. Therefore, the performance of proposed method is better than other existing methods in terms of accuracy for lower dimensionality and no feature selection.

\section{CONCLUSION}

In this paper, machine learning based blind JPEG classifier for detecting spliced images using noise and texture features has been proposed and implemented. The statistical differentiating features from original and spliced images have been extracted. Original images at QF100 and spliced images at various quality factors i.e. QF60, QF80, QF100 have been considered to train and test LIBSVM based classifier. The accuracy obtained demonstrated that proposed classifier gives better efficiency as compared to existing one. The proposed classifier may be extended to make an integrated forensic tool which can detect copy move, seam carving, steganography and other types of tampering in images.

\section{REFERENCES}

[1] Rocha, A., Scheirer, W., Bolt, T. and Golden stein, S. (2011) 'Vision of the unseen: current trends and challenges in digital image and video forensics', ACM Computer Survey, Vol. 43 No. 4, pp. 26:1-42.

[2] Birajdar, G. K. and Mankar, V. H. (2013) 'Digital image forgery detection using passive techniques: A survey’, Digital Investigation, 2013, Vol. 10 No.3, pp. 226-245.

[3] Li, X. (2002) 'Blind image quality assessment', Proceedings of 2002 International Conference on Image Processing, IEEE, Vol. 1, pp. I-I.

[4] Ascribes, I., Bayram, S., Memon, N., Ramkumar, M. and Sankur, B. (2004) 'A classifier design for detecting image manipulations', Proceeding of International Conference on Image Processing, IEEE, Vol. 4, pp. 2645-2648.

[5] Popescu, A.C. and Farid, H. (2004) 'Statistical tools for digital forensics', International Workshop on Information Hiding, pp. 128-147.
[6] Bayram, S., Avcıbaş, İ., Sankur, B. and Memon, N. (2006) 'Image manipulation detection', Journal of Electronic Imaging, Vol. 15 No. 4, pp. 041102-041102.

[7] Gou, H., Swaminathan, A. and Wu, M. (2007) 'Noise features for image tampering detection and steganalysis’, Proceedings of International Conference on Image Processing, IEEE, Vol. 6, pp. VI-97.

[8] Zhang, Z., Kang, J. and Ren, Y. (2008) 'An effective algorithm of image splicing detection', International Conference on Computer Science and Software Engineering, IEEE, Vol. 1, pp. 1035-1039.

[9] Mahdian, B. and Saic, S. (2009) 'Using noise inconsistencies for blind image forensics', Image and Vision Computing, Vol. 27, No. 10, pp.1497-1503.

[10] Wang, W., Dong, J. and Tan, T. (2009) 'Effective image splicing detection based on image chroma', Proceedings of 16th IEEE International Conference on Image Processing, pp. 1257-1260.

[11] Battisti, F., Carli, M. and Neri, A. (2012) 'Image forgery detection by means of no-reference quality metrics'. Proceeding of SPIE-Media Watermarking, Security, and Forensics.

[12] Ke, Y., Zhang, Q., Min, W. and Zhang, S. (2014) 'Detecting Image Forgery Based on Noise Estimation'. International Journal of Multimedia and Ubiquitous Engineering, 9(1), pp. 325-336.

[13] Liu, B. and Pun, C.M. (2015) 'Splicing Forgery Exposure in Digital Image by Detecting Noise Discrepancies', International Journal of Computer and Communication Engineering, 4(1), p.33.

[14] Shi, Y. Q., Chen, C. and Chen, W. (2007) 'A natural image model approach to splicing detection'. In: Proceedings of the 9th workshop on Multimedia \& security, pp: 51-62.

[15] He, Z., Lu, W., Sun, W. and Huang, J. (2012) 'Digital image splicing detection based on Markov features in DCT and DWT domain'. Pattern Recognition, 45(12): 4292-4299.

[16] Shen, X., Shi, Z. and Chen, H. (2016) 'Splicing image forgery detection using textural features based on the grey level cooccurrence matrices', IET Image Processing, 11(1):44-53.

[17] Dong, J. and Wang, W. (2011) 'CASIA tampered image detection evaluation database'. Available from: http://forensics.idealtest.org.

[18] Chang, C. C. and Lin, C. J. (2011) 'LIBSVM: a library for support vector machines’, ACM Transactions on Intelligent Systems and Technology (TIST), 2(3): 27. 